Military Technical College

Kobry Elkobbah,

Cairo, Egypt

May 27-29,2008

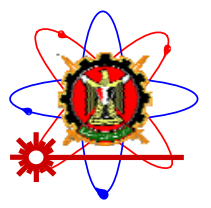

WP-3 $4^{\text {th }}$ International Conference on Mathematics and Engineering Physics (ICMEP-4)

\title{
Measurement of Glucose Nanoparticles Concentration in Plasma and Blood accurately using Laser Sheet Interferometry
}

\author{
H.El Ghandoor \\ Physics Department, Faculty of Science \\ Ain Shams University \\ Cairo-Egypt
}

\begin{abstract}
One of the major diseases in Egypt and the rest of the world is diabetes. In diabetic patients the synthesis and metabolism of glucose is abnormal that leads to abnormal blood glucose levels in the body tissues. Diabetic patients suffer form many acute and chronic symptoms and diseases. They are at high risk of heard disease, blindness, and reduced blood circulation in extremities often leading to ulcers, gangrenes and limb imputations. It is vital for diabetic patients to monitor blood glucose levels and take medication t control the glucose level in their body.

In this paper, a new technique developed by H.El Ghandoor, called the laser sheet method, will be applied to measure the optical properties of nanoparticles in blood plasma and blood. The technique involves relating the molar reflectivity of plasma to the glucose concentration. Comparing the measurement to the standard clinical method will do validation of the measurement.

Many types of glucose meters are commercially available. Glucose meters are a 3 billion dollar business worldwide. We would like to investigate a new technique for measuring the glucose level in the blood using laser refraction technique. If the technique works it will be the basis for a new instrument the Laser Interference Glucometery (LIG). We plan to originally measure blood glucose in the blood serum. If this is successful we plan to try the measurement on whole blood and possibly develop a non-invasive method for measuring the glucose level. The use of laser interference to determine the blood constituents may prove to be a very sensitive technique to determine blood and other biological fluid constituents. This laser interference technique might become an important accurate medical diagnostic tool in the future.
\end{abstract}

